The Astrophysical Journal, 346:193-200, 1989 November 1

(C) 1989. The American Astronomical Society. All rights reserved. Printed in U.S.A.

\title{
FURTHER STUDIES OF THE ROLE OF DENSE MOLECULAR CLOUDS AROUND OUTFLOW SOURCES
}

\author{
LOURDES Verdes-Montenegro AND José M. TORRELleS \\ Instituto de Astrofisica de Andalucía, CSIC \\ LUIS F. RODRÍGUEZ ${ }^{1}$ \\ Harvard-Smithsonian Center for Astrophysics \\ Guillem Anglada, ${ }^{2}$ Rosario López, ${ }^{2}$ AND Robert Estalella ${ }^{2}$ \\ Departament de Física de l'Atmosfera, Astronomia i Astrofísica, UB \\ JoRge CANTÓ \\ Instituto de Astronomía, UNAM \\ AND \\ Paul T. P. Ho \\ Harvard-Smithsonian Center for Astrophysics \\ Received 1989 January 11; accepted 1989 April 24
}

\begin{abstract}
We observed with the $37 \mathrm{~m}$ telescope of the Haystack Observatory the $(J, K)=(1,1)$ and $(2,2)$ ammonia inversion transitions toward six regions with active star formation and evidence of outflows: AFGL 5142, HH 33/40, AFGL 5157, AFGL 6366S, HHL 73, and S140N. $(J, K)=(1,1)$ ammonia emission was detected and mapped toward all sources except for $\mathrm{HH} 33 / 40$, while $(J, K)=(2,2)$ ammonia emission was detected toward AFGL 5157 and S140N. We derived rotational temperatures of $T_{R}(2,2 ; 1,1) \leq 17 \mathrm{~K}$ in all cases. We also searched for $\mathrm{H}_{2} \mathrm{O}$ masers, and intense emission $\left(S_{v} \geq 40 \mathrm{Jy}\right)$ was detected toward the AFGL 5142 and AFGL 5157 ammonia cores. It is clear that the dense ammonia gas is closely associated with the star formation activities, since the ammonia cores in all cases peak close to the centers of activity. In particular, the AFGL 5157 ammonia condensation coincides with the geometrical center of a bipolar molecular outflow suggesting that the exciting source is embedded in the ammonia core. In contrast, the molecular outflow in the AFGL $6366 \mathrm{~S}$ region is located at the southeast edge of the ammonia condensation, suggesting that the exciting source is outside the ammonia core and that the morphology of the outflow may be influenced by the interaction with the dense ambient gas.
\end{abstract}

Subject headings: interstellar: molecules - masers - nebulae: general — stars: pre-main-sequence

\section{INTRODUCTION}

With the discovery of molecular outflows in Orion (Zuckerman, Kuiper, and Rodríguez Kuiper 1976; Kwan and Scoville 1976), and elsewhere (Rodríguez et al. 1982; Lada 1985), the earliest stage of stellar evolution has become associated with strong mass outflows. An effort has been made by our group and others to study the physical properties and the role of the high-density gas around sources with molecular outflows. Observations of molecular tracers of high-density gas, such as ammonia $\left(\mathrm{NH}_{3}\right)$ and carbon monosulfide (CS), have established that the suspected exciting sources of the molecular outflows, as indicated by infrared sources (IRS), $\mathrm{H}_{2} \mathrm{O}$ and $\mathrm{OH}$ masers, and compact $\mathrm{H}$ II regions, are usually engulfed in high-density molecular condensations (Torrelles et al. 1983, 1985, 1986a; Schwartz, Waak, and Smith 1983; Little et al. 1985; Heyer et al. 1986; Takano et al. 1987; Myers et al. 1988; Marcaide et al. 1988). Thus, for example, Anglada et al. (1989) have been able to use the statistical spatial correlation of high-density gas with the activity centers of outflows to help localize the position of undetected power sources. In addition, the high-density gas is often perturbed by the pressure of the

\footnotetext{
${ }^{1}$ On sabbatical leave from Instituto de Astronomía, UNAM.

${ }^{2}$ Grup d'Astrofísica, Societat Catalana de Física, IEC.
}

stellar wind and by the stellar photons. Evidences for these effects are high-velocity wings in the $\mathrm{NH}_{3}$ and $\mathrm{CS}$ line emission (Thronson and Lada 1984; Takano et al. 1985; Torrelles et al. $1987 b$ ), the presence of broad ammonia lines associated with molecular outflows (Myers et al. 1988), and local heating effects on the molecular gas (Torrelles et al. 1986b, 1989; Takano 1986). This perturbation of the ambient gas has also been used successfully to aid in identifying the exciting sources.

Another important aspect of the dense molecular cores is their relation to the focusing mechanism of the observed bipolar outflows. A perpendicular alignment between elongated high-density structures of interstellar dimensions $\left(\sim 10^{17}\right.$ $\mathrm{cm}$ ), and the bipolar outflows, suggested that interstellar toroids may collimate an initially isotropic outflow (e.g., Torrelles et al. 1983, 1985; Schwartz, Waak, and Smith 1983; Little et al. 1985; Kawabe et al. 1989; Marcaide et al. 1988). This appeared consistent with theoretical models (Barral and Cantó 1981; Königl 1982; Boss 1987). However, there are two main objections to this interpretation: (1) in some cases, such as L1551, different molecular tracers of high-density gas show different morphologies (Torrelles et al. 1983; Menten and Walmsley 1985; Kaifu et al. 1984; Moriarty-Schieven et al. 1987), and (2) there is observational evidence that collimation processes are also acting at smaller, circumstellar scales, e.g., optical jets (Mundt 1986), perhaps due to a nonuniform cir- 
TABLE 1

SOURCES MAPPED IN $\mathrm{NH}_{3}(1,1)$

\begin{tabular}{|c|c|c|c|c|c|c|}
\hline \multirow[b]{2}{*}{ SOURCE } & \multicolumn{2}{|c|}{ Map Central Position } & \multirow{2}{*}{$\begin{array}{c}\text { Nature of Source } \\
\text { NEAR CENTRAL } \\
\text { Position }\end{array}$} & \multirow{2}{*}{$\begin{array}{c}\text { TYPICAL } 1 \sigma \\
\text { SENSITIVITY } \\
\text { OBTAINED } \\
(\mathrm{K})\end{array}$} & \multirow{2}{*}{$\begin{array}{c}\mathrm{NH}_{3}(1,1) \\
\text { DETECTED? }\end{array}$} & \multirow{2}{*}{$\begin{array}{c}\text { NUMBER OF } \\
\text { OBSERVED } \\
\text { POSITIONS }\end{array}$} \\
\hline & $\alpha(1950)$ & $\delta(1950)$ & & & & \\
\hline AFGL 5142 ${ }^{\mathrm{a}}$ & $05^{\mathrm{h}} 27^{\mathrm{m}} 27^{\mathrm{s}} 6$ & $33^{\circ} 45^{\prime} 37^{\prime \prime}$ & Molecular outflow and infrared source & 0.06 & Yes & 14 \\
\hline HH $33 / 40^{b}$.. & $0532 \quad 53.0$ & -061956 & Double HH object; infrared source & 0.03 & No & 5 \\
\hline AFGL $5157^{\mathrm{a}}$ & 053435.7 & 315824 & Molecular outflow and infrared source & 0.04 & Yes & 28 \\
\hline AFGL $6366 \mathrm{~S}^{\mathrm{a}} \quad \ldots \ldots \ldots \ldots$ & 060546.5 & 213116 & Molecular outflow and infrared source & 0.04 & Yes & 32 \\
\hline HHL $73^{c} \ldots \ldots \ldots \ldots \ldots \ldots$ & 214318.0 & 471900 & $\mathrm{H}_{2} \mathrm{O}$ maser; Herbig-Haro-like object & 0.04 & Yes & 43 \\
\hline $\mathrm{S} 140 \mathrm{~N}^{\mathrm{d}} \ldots \ldots \ldots \ldots \ldots \ldots$ & 221751.5 & 631750 & Molecular outflow and infrared source & 0.04 & Yes & 36 \\
\hline
\end{tabular}

a Snell et al. 1988.

${ }^{b}$ Mundt et al. 1984; Cohen and Schwartz 1987.

c Gyulbudaghian, Rodríguez, and Mendoza-Torres 1987.

d Fukui et al. 1986.

cumstellar environment $(\leq 1000 \mathrm{AU}$; e.g., Cantó, TenorioTagle, and Różyczka 1988), or circumstellar accretion disks (e.g., Hartmann and Kenyon 1987). Whether the jet phenomenon can actually power the molecular outflow has been questioned by Sarcander, Neckel, and Elsässer (1985), who show that the momentum rate observed in the jet of L1551-IRS 5 is only about $1 \%$ of what is needed to drive the molecular outflow. It is possible that focusing and collimation processes operate from circumstellar dimensions (focusing the jet) to interstellar dimensions (focusing the molecular outflow) (Rodríguez et al. 1986).

In this paper we present the results of an observational ammonia survey toward six regions with recently reported gas outflow, traced by the presence of molecular outflows (AFGL 5142, AFGL 5157, AFGL 6366S, Snell et al. 1988; S140N, Fukui et al. 1986), and Herbig-Haro objects (HHL 73, Gyulbudaghian, Rodríguez, and Mendoza-Torres 1987; HH 33/40, Mundt et al. 1984, Cohen and Schwartz 1987). Three of these regions, AFGL 5142, AFGL 5157, and AFGL 6366S, have been studied by Snell et al. (1988) in a systematic CO observational program, searching for molecular outflows toward bright far-infrared sources. The three sources selected from Snell et al. (1988) are those with a bipolar pattern.

This ammonia study enlarges the sample of outflow sources studied with molecular tracers of high-density gas. In $\S$ II we describe the observations, in $\S$ III we discuss the individual sources, and we present in $\S$ IV our main conclusions.

\section{OBSERVATIONS AND DATA ANALYSIS}

Observational data were obtained with the $37 \mathrm{~m}$ radio telescope at Haystack Observatory ${ }^{3}$ in Westford, Massachusetts, during 1988 February and May. We selected the $(J, K)=(1,1)$ and $(2,2)$ inversion transitions of ammonia for the study of the molecular condensations around the outflow sources. At the frequency of these transitions $(\sim 23.7 \mathrm{GHz})$ the beam size is $1^{\prime} \cdot 4$, and the beam efficiency at an elevation of $45^{\circ}$ is 0.32 . We estimate an rms pointing accuracy of $\sim 15^{\prime \prime}$. We used a cooled K-band maser receiver and a 1024 channel digital autocorrelation spectrometer with an effective bandwidth of $6.67 \mathrm{MHz}$. After Hanning weighting, the velocity resolution was $\sim 0.2 \mathrm{~km}$ $\mathrm{s}^{-1}$. The observations were made using the position switching mode, with typically 10 minutes of total integration time (ON plus OFF). The spectra were corrected for elevation-dependent gain variation and atmospheric attenuation. The ammonia observations were made under good atmospheric conditions, with typical system temperatures of $\sim 80 \mathrm{~K}$.

\footnotetext{
${ }^{3}$ Radio astronomy at Haystack Observatory of the Northeast Radio Observatory Corporation is supported by the National Science Foundation.
}

We observed six sources, and began by making a five-point full beam separation $\mathrm{NH}_{3}(1,1)$ map centered at the position of the suspected activity centers of the outflows (these positions are indicated in Table 1). We detected $\mathrm{NH}_{3}(1,1)$ emission in five of the sources. Maps of the ammonia emission with typically $\sim 30$ positions were made for the detected sources in order to delimit the ammonia emission. The total integration time per position was, typically, 40 minutes, and a sensitivity per spectral channel of $0.04 \mathrm{~K}$ was reached.

In Table 2 we give the $\mathrm{NH}_{3}(1,1)$ line parameters observed at the positions of the emission peak of the mapped sources. The spectra obtained at these positions are shown in Figure 1. In Figures 3,5,6,7, and 9, we show the contour maps of the peak

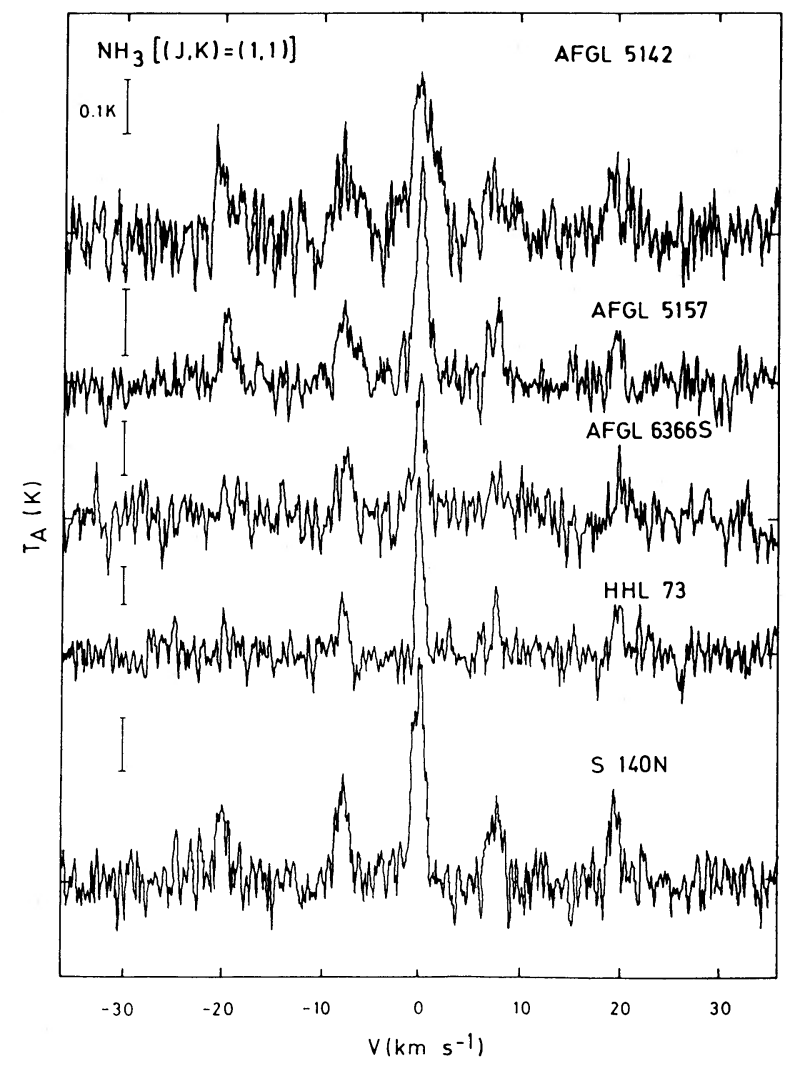

Fig. 1.-Observed spectra of the $(J, K)=(1,1)$ inversion transition line of $\mathrm{NH}_{3}$ at the positions given in Table 2 . The horizontal axis is the radial velocity with respect to the center of the main line $(1,1 ; \mathrm{m})$. We show the central $87 \%$ of the $6.67 \mathrm{MHz}$ bandwidth. The vertical bar shown in each spectrum is equivalent to $0.1 \mathrm{~K}$ in antenna temperature $\left(T_{A}\right)$. 
TABLE 2

Line Parameters at Selected Positions of the Sources Detected in $\mathrm{NH}_{3}(1,1)^{\mathrm{a}}$

\begin{tabular}{|c|c|c|c|c|c|c|}
\hline \multirow[b]{2}{*}{ SOURCE } & \multicolumn{2}{|c|}{ Position } & \multirow{2}{*}{$\begin{array}{c}T_{A}(1,1 ; \mathrm{m}) \\
(\mathrm{K})\end{array}$} & \multirow{2}{*}{$\frac{T_{A}(1,1 ; \mathrm{m})}{T_{A}(1,1 ; \text { is })}$} & \multirow{2}{*}{$\begin{array}{c}\Delta V(1,1 ; \mathrm{m}) \\
\left(\mathrm{km} \mathrm{s}^{-1}\right)\end{array}$} & \multirow{2}{*}{$\begin{array}{c}V_{\mathrm{LSR}}(1,1 ; \mathrm{m}) \\
\left(\mathrm{km} \mathrm{s}^{-1}\right)\end{array}$} \\
\hline & $\alpha(1950)$ & $\delta(1950)$ & & & & \\
\hline AFGL 5142 & $05^{\mathrm{h}} 27^{\mathrm{m}} 27^{\mathrm{s}} .6$ & $33^{\circ} 45^{\prime} 37^{\prime \prime}$ & $0.26 \pm 0.01$ & $2.6 \pm 0.2$ & $2.86 \pm 0.14$ & $-3.43 \pm 0.06$ \\
\hline AFGL 5157. & $\begin{array}{lll}05 & 34 & 35.7\end{array}$ & 315824 & $0.30 \pm 0.01$ & $3.3 \pm 0.3$ & $1.50 \pm 0.05$ & $-18.04 \pm 0.02$ \\
\hline AFGL 6366S $\ldots \ldots \ldots \ldots$ & $0605 \quad 40.1$ & 213116 & $0.24 \pm 0.02$ & $\geq 1.6^{\mathrm{b}}$ & $1.04 \pm 0.13$ & $2.46 \pm 0.05$ \\
\hline HHL $73 \ldots \ldots \ldots \ldots \ldots$ & $2143 \quad 13.6$ & 471945 & $0.48 \pm 0.02$ & $3.1 \pm 0.3$ & $0.73 \pm 0.04$ & $3.87 \pm 0.02$ \\
\hline 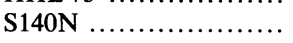 & 221758.1 & 631750 & $0.39 \pm 0.01$ & $2.6 \pm 0.1$ & $1.33 \pm 0.05$ & $-8.81 \pm 0.02$ \\
\hline
\end{tabular}

a Obtained from Gaussian fits to the observed spectra. The notation in the column headings $(1,1 ; \mathrm{m})$ refers to the main hyperfine line, and $(1,1$; is) refers to the inner satellite lines.

${ }^{b}$ In this source no Gaussian profiles were fitted to the inner satellite lines.

TABLE 3

$\mathrm{NH}_{3}(2,2)$ OBSERVATIONS ${ }^{\mathrm{a}}$

\begin{tabular}{|c|c|c|c|c|}
\hline Source & $\begin{array}{c}\text { Total } \\
\text { Integration } \\
\text { Time } \\
\text { (ON+OFF) } \\
\text { (hr) }\end{array}$ & $\begin{array}{c}\text { Typical } 1 \sigma \\
\text { Sensitivity } \\
\text { Obtained } \\
\text { (K) }\end{array}$ & $\begin{array}{l}\mathrm{NH}_{3}(2,2) \\
\text { Detected? }\end{array}$ & $\begin{array}{c}T_{A}(2,2 ; \mathrm{m}) \\
(\mathrm{K})\end{array}$ \\
\hline AFGL 5142 & 1.0 & 0.03 & No & $\leq 0.09^{\mathrm{b}}$ \\
\hline AFGL 5157 & 1.8 & 0.02 & Yes & $0.10 \pm 0.01^{\mathrm{c}}$ \\
\hline AFGL 6366S $\ldots \ldots \ldots \ldots$ & 1.2 & 0.03 & No & $\leq 0.09^{\mathrm{b}}$ \\
\hline HHL $73 \ldots \ldots \ldots \ldots \ldots$ & 1.0 & 0.04 & No & $\leq 0.12^{\mathrm{b}}$ \\
\hline S140N $\ldots \ldots \ldots \ldots \ldots \ldots$ & 1.0 & 0.03 & Yes & $0.13 \pm 0.01^{\mathrm{c}}$ \\
\hline
\end{tabular}

${ }^{a} \mathrm{NH}_{3}(2,2)$ observations toward the positions given in Table 2 .

${ }^{6}$ Upper limit $(3 \sigma)$.

c Obtained from Gaussian fit to the observed spectra.

antenna temperature of the main line of the $(1,1)$ ammonia transition $\left[T_{A}(1,1 ; \mathrm{m})\right]$. Several indicators of star formation are also shown in the maps.

We also observed the $(J, K)=(2,2)$ ammonia transition toward the positions given in Table 2 in order to obtain the rotational temperature of the ammonia condensations. We detected this line in two of the sources, with upper limits in the other three (Table 3). The $\mathrm{NH}_{3}(2,2)$ spectra obtained are shown in Figure 2.

In Table 4 we give the parameters of the ammonia condensations: size $\left(l_{a} \times l_{b}\right)$, optical depth $[\tau(1,1 ; \mathrm{m})]$, excitation temperature $\left[T_{\mathrm{ex}}(1,1)\right]$, rotational temperature $\left[T_{R}(2,2 ; 1,1)\right]$, hydrogen volume density $\left[n\left(\mathrm{H}_{2}\right)\right]$, mass $(M)$, hydrogen column density $\left[N\left(\mathrm{H}_{2}\right)\right]$, and visual extinction $\left(A_{V}\right)$. These parameters have been derived as indicated in the footnotes to Table 4.

Finally we searched for $\mathrm{H}_{2} \mathrm{O}$ maser emission at $22.2 \mathrm{GHz}$ toward three of the sources detected in ammonia (AFGL 5142, AFGL 5157, AFGL 6366S), in order to obtain additional information on the location of the exciting source. We used a cooled K-band maser receiver and a 512 channel digital autocorrelator spectrometer with an effective bandwidth of 13.3 MHz. We made a seven-point map around the positions given in Table 1, and detected two new strong masers toward AFGL 5142 and AFGL $5157\left(S_{v} \geq 40 \mathrm{Jy} ;\right.$ Fig. 4). The upper limit (3 $\left.\sigma\right)$

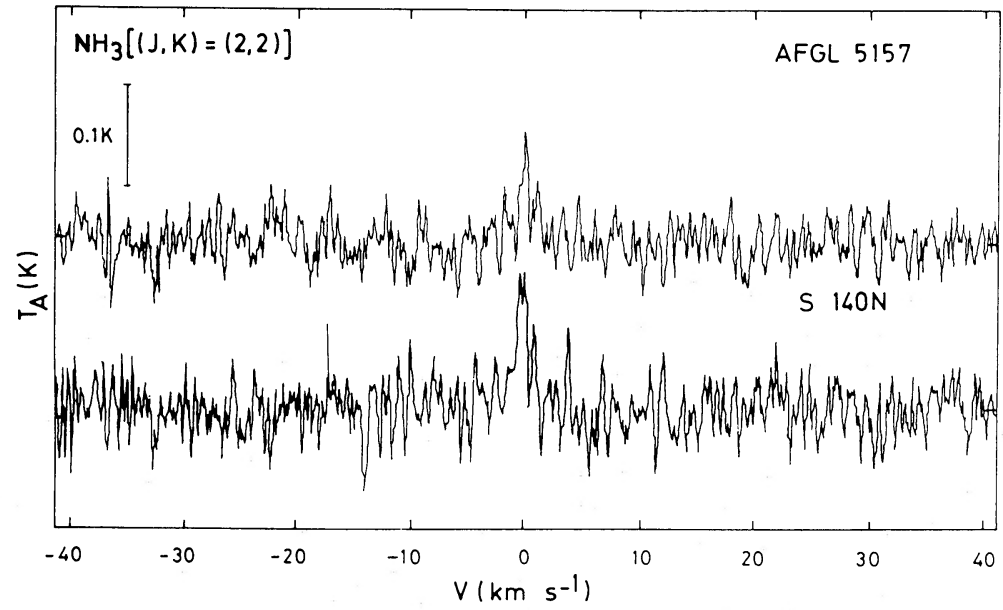

Fig. 2.- Observed spectra of the $(J, K)=(2,2)$ inversion transition line of $\mathrm{NH}_{3}$ toward AFGL 5157 and S140N (positions are given in Table 2). The horizontal axis is the radial velocity with respect to the center of the main line $(2,2 ; \mathrm{m})$. The vertical bar is equivalent to $T_{A}=0.1 \mathrm{~K}$. 
TABLE 4

Parameters of the Ammonia Condensations

\begin{tabular}{|c|c|c|c|c|c|c|c|c|c|c|}
\hline Source & $\begin{array}{c}\text { Distance } \\
\text { (pc) }\end{array}$ & $\begin{array}{c}\text { Angular Size }^{\mathrm{a}} \\
l_{a} \times l_{b} \\
(\operatorname{arcmin})\end{array}$ & $\begin{array}{c}\mathrm{Size}^{\mathrm{a}} \\
l_{a} \times l_{b} \\
(\mathrm{pc})\end{array}$ & $\tau(1,1 ; \mathrm{m})^{\mathbf{b}}$ & $\begin{array}{c}T_{\mathrm{ex}}(1,1)^{\mathrm{c}} \\
(\mathrm{K})\end{array}$ & $\begin{array}{c}T_{R}(2,2 ; 1,1)^{\mathrm{d}} \\
(\mathrm{K})\end{array}$ & $\begin{array}{l}n\left(\mathbf{H}_{2}\right)^{e} \\
\left(\mathrm{~cm}^{-3}\right)\end{array}$ & $\begin{array}{c}M^{\mathrm{f}} \\
\left(M_{\odot}\right)\end{array}$ & $\begin{array}{l}N\left(\mathbf{H}_{2}\right)^{\mathrm{g}} \\
\left(\mathrm{cm}^{-2}\right)\end{array}$ & $\begin{array}{c}A_{V}^{\mathrm{h}} \\
\text { (mag) }\end{array}$ \\
\hline AFGL 5142 & $1800^{i}$ & $\sim 2.3 \times 2.3$ & $\sim 1.2 \times 1.2$ & $1.0 \pm 0.3$ & 4 & $\leq 16$ & $\sim 4 \times 10^{3}$ & $\sim 200$ & $7 \times 10^{22}$ & 70 \\
\hline AFGL $5157 \ldots \ldots \ldots \ldots$ & $1800^{i}$ & $\sim 1.7 \times 1.7$ & $\sim 0.9 \times 0.9$ & $0.2 \pm 0.2$ & 8 & $\sim 17$ & $\sim 2 \times 10^{4}$ & $\sim 400$ & $8 \times 10^{21}$ & 8 \\
\hline AFGL $6366 \mathrm{~S} \ldots \ldots \ldots \ldots$ & $1500^{\mathrm{i}}$ & $\sim 7.1 \times 2.6$ & $\sim 3.1 \times 1.1$ & $\leq 3.3$ & $4^{j}$ & $\leq 16^{\mathrm{j}}$ & $\sim 4 \times 10^{3}$ & $\sim 650$ & $2 \times 10^{22 j}$ & 20 \\
\hline HHL $73 \ldots \ldots \ldots \ldots \ldots$ & $900^{k}$ & $\sim 4.7 \times 2.2$ & $\sim 1.2 \times 0.6$ & $0.4 \pm 0.3$ & 7 & $\leq 15$ & $\sim 3 \times 10^{4}$ & $\sim 500$ & $1 \times 10^{22}$ & 10 \\
\hline S140N ............... & $900^{1}$ & $\sim 2.2 \times 1.7$ & $\sim 0.6 \times 0.4$ & $1.0 \pm 0.2$ & 5 & $\sim 15$ & $\sim 6 \times 10^{3}$ & $\sim 20$ & $2 \times 10^{22}$ & 20 \\
\hline
\end{tabular}

a FWHM of the ammonia emission.

b Derived from $T_{A}(1,1 ; \mathrm{m}) / T_{A}(1,1 ;$ is) (Ho and Townes 1983).

c A filling factor of unity is assumed.

d Calculated from the ratio $T_{A}(2,2 ; \mathrm{m}) / T_{A}(1,1 ; \mathrm{m})$ (Ho and Townes 1983).

e Derived from the two-level model (Ho and Townes 1983) assuming $T_{K}=T_{R}(2,2 ; 1,1)$ except for AFGL 5142, AFGL 6366S and HHL 73, where, given the

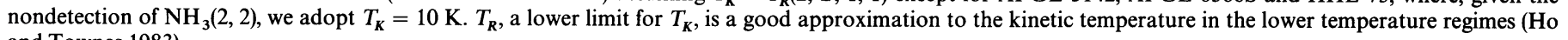
and Townes 1983).

A sphere of radius $\frac{1}{2}\left(l_{a} \times l_{b}\right)^{1 / 2}$ is assumed.

${ }^{\mathrm{g}}$ LTE for the rotational states of ammonia at $T_{K}$, and an abundance $\left[\mathrm{NH}_{3} / \mathrm{H}_{2}\right]=10^{-8}($ Herbst and Klemperer 1973$)$ have been assumed.

${ }^{\text {h }}$ From $\left[A_{V} / \mathrm{mag}\right]=10 \times\left[N\left(\mathrm{H}_{2}\right) / 10^{22} \mathrm{~cm}^{-2}\right]($ Spitzer 1978).

i Snell et al. 1988.

$\mathrm{j} \tau(1,1 ; \mathrm{m})=1$ is assumed.

k Elias 1978.

${ }^{1}$ Crampton and Fisher 1974.

for AFGL 6366S is $\sim 1 \mathrm{Jy}$. Water maser emission has been reported previously for HHL 73 (Gyulbudaghian, Rodríguez, and Mendoza-Torres 1987).

\section{INDIVIDUAL SOURCES}

a) AFGL 5142 (IRAS 05274 + 3345)

The center of symmetry of the red and blue wings of the $\mathrm{CO}$ bipolar outflow coincides with AFGL 5142. We started our ammonia map at this position. In Figure 3 we show a superposition of the $\mathrm{NH}_{3}$ and bipolar outflow maps. The detected high-density structure is slightly resolved, and it peaks at the AFGL 5142 position. The $\mathrm{NH}_{3}(1,1 ; \mathrm{m})$ spectrum at the central position shows a broad line $\left(\Delta V \sim 2.9 \mathrm{~km} \mathrm{~s}^{-1}\right)$, suggesting the existence of different velocity components unresolved by our beam and/or a strong perturbation of the molecular gas produced by AFGL 5142. We detected a strong $\mathrm{H}_{2} \mathrm{O}$ maser with five velocity components at $V_{\mathrm{LSR}}=-13.4,-11.1,-4.8,-1.6$, and $0.4 \mathrm{~km} \mathrm{~s}^{-1}$ (Fig. 4). For the strongest components $\left(S_{v} \sim 43\right.$ $\mathrm{Jy} ; V_{\mathrm{LSR}} \sim 0.4 \mathrm{~km} \mathrm{~s}^{-1}$ ) we estimate a position of $\alpha(1950)=$ $05^{\mathrm{h}} 27^{\mathrm{m}} 30^{\mathrm{s}} 8, \delta(1950)=33^{\circ} 45^{\prime} 12^{\prime \prime}\left( \pm 40^{\prime \prime}\right)$, inside the ammonia clump.

These results give support to the identification of AFGL 5142 as the powering source of the $\mathrm{CO}$ bipolar outflow. However, higher angular resolution observations of the ammonia clump are needed to delimit its shape and orientation with respect to the bipolar outflow, and to clarify the role of the high-density structure in the collimation process of the bipolar outflow.

\section{b) AFGL 5157 (IRAS $05345+3157)$}

This IRAS source seems to be spatially associated with NGC $1985 \quad\left[\alpha(1950)=05^{\mathrm{h}} 34^{\mathrm{m}} 32^{\mathrm{s}} \mathrm{s}, \quad \delta(1950)=+31^{\circ} 57^{\prime} 36^{\prime \prime}\right.$ $\left( \pm 10^{\prime \prime}\right)$; Higgs 1971], which has been identified as a reflection nebula with a central F1 star (Sabbadin and Hamzaoglu 1981; Lutz and Kaler 1983; Acker et al. 1987). However, they appear to be distinct objects, since AFGL 5157 is a very red embedded stellar object, probably a young B star (Snell et al. 1988). Snell

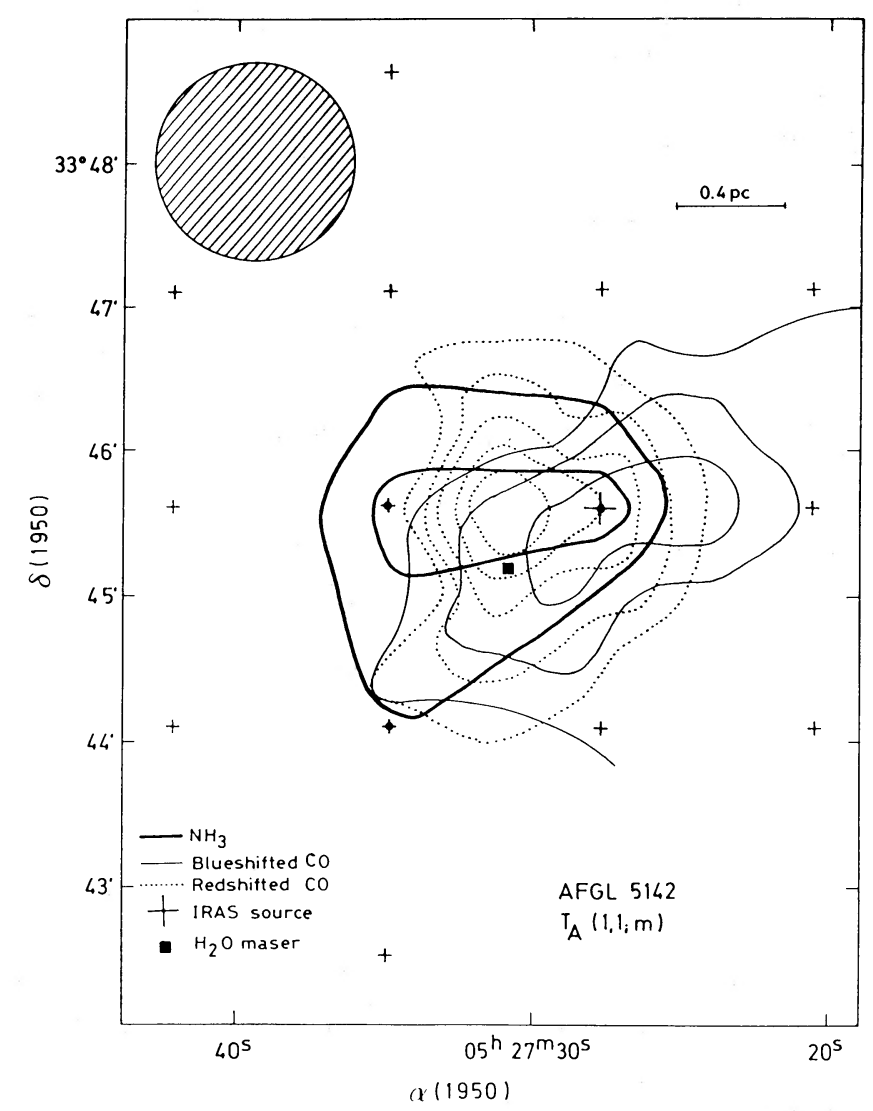

FIG. 3.-Contour map of the peak antenna temperature of the main line of the $(J, K)=(1,1)$ ammonia transition for AFGL 5142 (thick line; this paper), superposed on the contours of the CO bipolar outflow (Snell et al. 1988). Ammonia contours are $0.13,0.18$, and $0.23 \mathrm{~K}$. Small plus signs indicate the observed positions, and dots denote the positions where $\mathrm{NH}_{3}(1,1)$ was detected. The large plus sign denotes the position of the IRAS source. Its positional error is $\sim \pm 20^{\prime \prime}$. We indicate the position of the detected $\mathrm{H}_{2} \mathrm{O}$ maser (this paper, Fig. 4) by a filled square. 


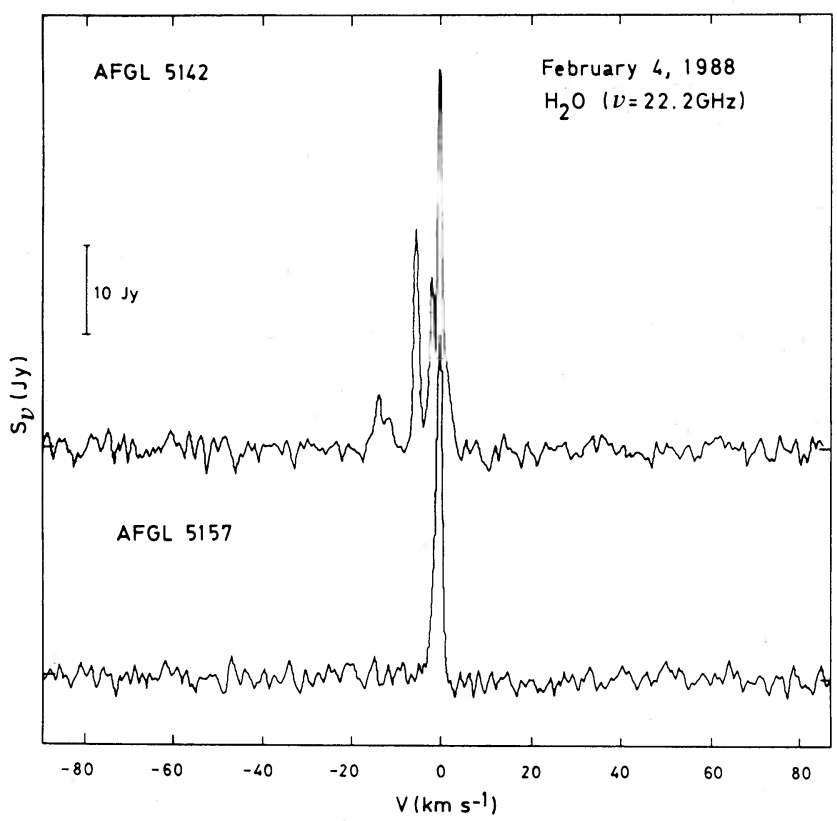

FIG. 4.-Observed spectra of the two new detected $\mathrm{H}_{2} \mathrm{O}$ masers toward AFGL 5142 and AFGL 5157 (this paper). The $\mathrm{H}_{2} \mathrm{O}$ maser in AFGL 5142 shows five velocity components at $V_{\mathrm{LSR}}=-13.4,-11.1,-4.8,-1.6$, and 0.4 $\mathrm{km} \mathrm{s}^{-1}$. The brightest $\left(V_{\mathrm{LSR}}=0.4 \mathrm{~km} \mathrm{~s}^{-1}\right)$ has a flux density $S_{\mathrm{v}} \sim 43 \mathrm{Jy}$. AFGL 5157 has just one velocity component at $V_{\mathrm{LSR}}=-20 \mathrm{~km} \mathrm{~s}^{-1}\left(S_{v} \sim 39 \mathrm{Jy}\right)$. The horizontal axis is the radial velocity with respect to the velocity of the brightest component of each source.

et al. (1988) have detected a clear bipolar molecular outflow located $1^{\prime}$ northeast of AFGL 5157.

We centered our $\mathrm{NH}_{3}(1,1)$ map at the geometrical center of the bipolar outflow (Table 1). We detected an ammonia clump barely resolved, peaking at that position (Fig. 5). $\mathrm{NH}_{3}(2,2)$

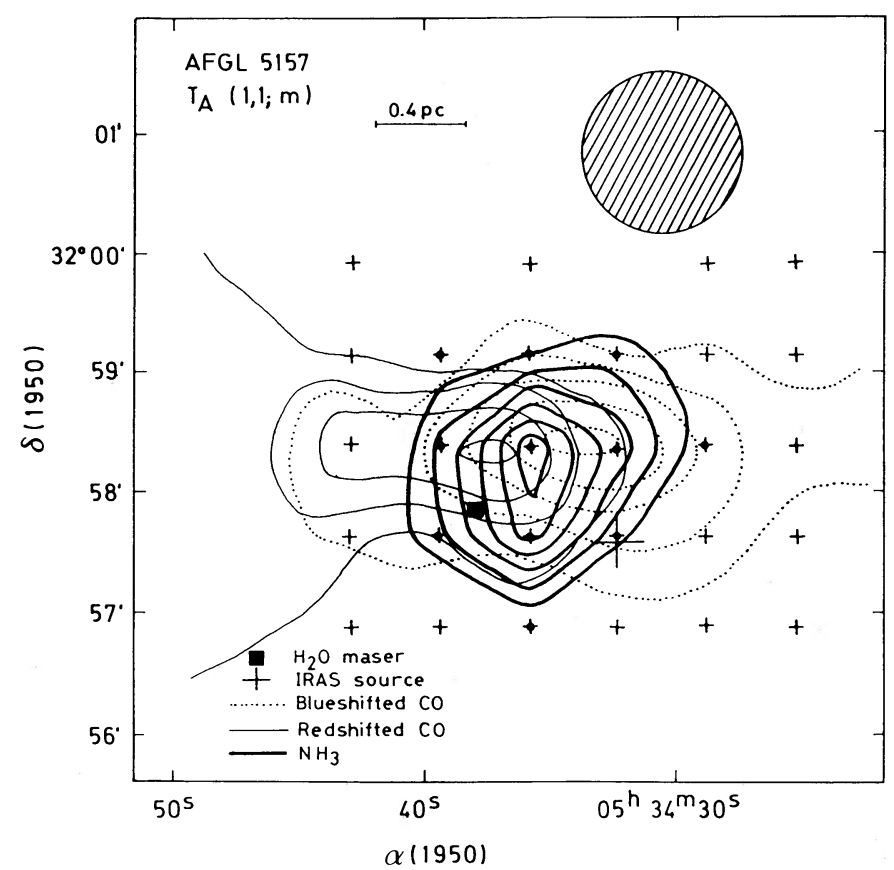

Fig. 5. - Same as Fig. 3, but for AFGL 5157. Ammonia contours are 0.13, $0.16,0.19,0.22$, and $0.25 \mathrm{~K} .\left[T_{A}(1,1 ; \mathrm{m})\right.$ contours and $\mathrm{H}_{2} \mathrm{O}$ maser, this paper; CO bipolar outflow, Snell et al. 1988]. The positional error of the IRAS source is $\sim \pm 20^{\prime \prime}$. emission was detected toward the peak of the $\mathrm{NH}_{3}(1,1)$ emission (Fig. 2; Table 3). From these data we estimate a rotational temperature between the $(2,2)$ and $(1,1)$ metastable states of $T_{R}(2,2 ; 1,1) \simeq 17 \mathrm{~K}$. This temperature is higher than the temperature observed in the dense cores of dark clouds $(\sim 10 \mathrm{~K}$; Myers and Benson 1983), where the cosmic rays are the main heating mechanism (Goldsmith and Langer 1978). Therefore, a local heating mechanism is required. We also detected a strong $\mathrm{H}_{2} \mathrm{O}$ maser $\left(S_{v} \sim 39 \mathrm{Jy}\right)$ at $\alpha(1950)=05^{\mathrm{h}} 34^{\mathrm{m}} 37^{\mathrm{s}} .9$, $\delta(1950)=+31^{\circ} 57^{\prime} 51^{\prime \prime}\left( \pm 10^{\prime \prime}\right)$ and $V_{\mathrm{LSR}}=-20 \mathrm{~km} \mathrm{~s}^{-1}$ (Fig. 4). These results suggest that a more promising candidate for the powering source of the outflow may be located at the position of the ammonia peak. This exciting source could also be responsible for the observed local heating effect and for the $\mathrm{H}_{2} \mathrm{O}$ maser emission. A search for near- and far-infrared emission at this position could give an important clue for the identification of the exciting source. Finally, observations with higher angular resolution than ours are needed in order to clarify the role of the detected high-density structure in the focusing of this bipolar outflow. In particular, given the spatial coincidence of the detected compact ammonia clump with the activity center of the outflow, it is important to determine whether the ammonia core, when observed with higher angular resolution, presents an elongated structure perpendicular to the bipolar outflow as in other cases reported in the literature.

\section{c) $A F G L 6366 S(I R A S 06056+2131)$}

Snell et al. (1988) detected a CO bipolar outflow with a low degree of collimation displaced $\sim 1.3$ southeast from AFGL $6366 \mathrm{~S}$. We started our ammonia map at the geometrical center of the molecular outflow. We detected an ammonia condensation elongated in the southwest-northeast direction. The ammonia emission continues to the northeast, outside the mapped region (Fig. 6). The maximum of the ammonia emission coincides with AFGL 6366S. However, the molecular outflow is situated at the southeast edge of the ammonia core. To our knowledge, this is the first case in which such a peculiar spatial disposition between the ammonia condensation and the bipolar molecular outflow is found. This observational result suggests that it is unlikely for AFGL 6366S to be the powering source of the bipolar molecular outflow. If the exciting object is located near the outflow center, at the southeast boundary of the high-density gas, the ammonia condensation would not play a direct role in the collimation processes. However, it is possible that the high-velocity gas associated with the exciting object has been deflected or swept up from the high-density gas. This interaction could originate, at the border of the ammonia clump, the observed molecular outflow. Torrelles et al. (1987a) have discussed the formation of high-velocity molecular gas as a result of the interaction of an outflow with the surface of a cloud.

\section{d) $\mathrm{HHL} 73$}

There are several star formation tracers in this region: a Herbig-Haro-like object (HHL 73), where we centered our ammonia map; an $\mathrm{H}_{2} \mathrm{O}$ maser (Gyulbudaghian, Rodríguez, and Mendoza-Torres 1987); and an IRAS source. A CO outflow has been detected (Levreault 1988) at the position of the FU Orionis star Elias 1-12 (Elias 1978), located $\sim 20^{\prime}$ east of HHL 73. We will adopt for the HHL 73 region a distance of $0.9 \mathrm{kpc}$, the value given by Elias (1978) for this FU Orionis star.

Our observations show two ammonia clumps (Fig. 7). The main clump is elongated in the southeast-northwest direction. 


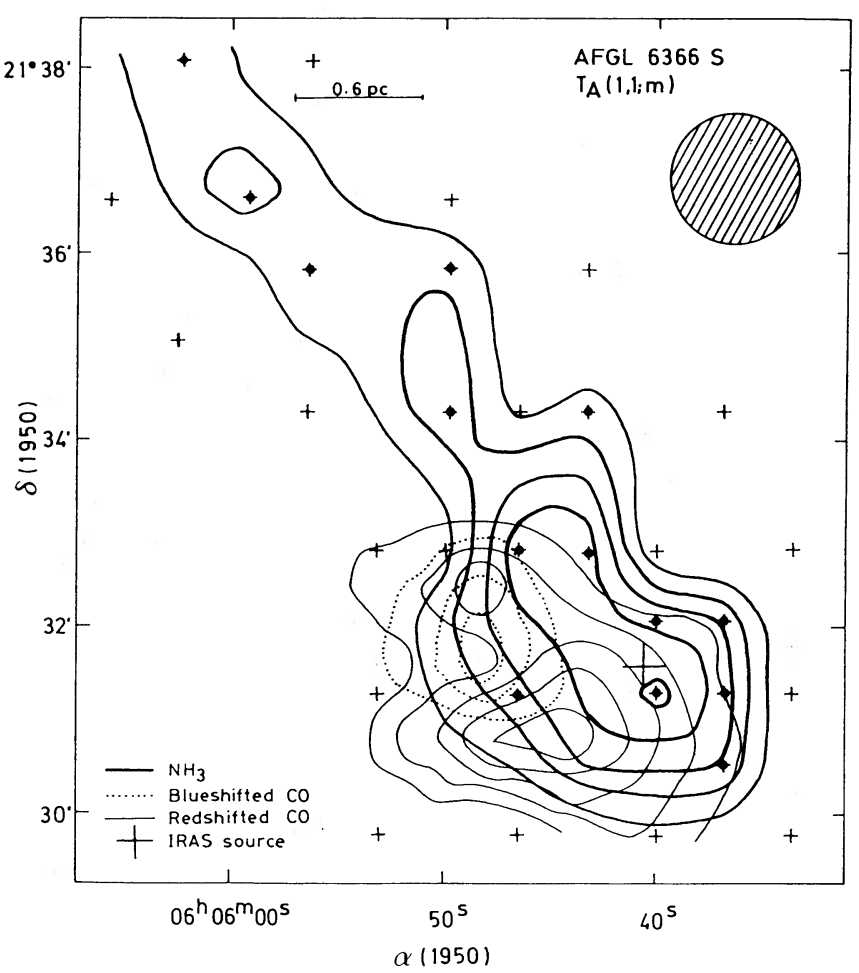

Fig. 6. - Same as Fig. 3, but for AFGL 6366S. Ammonia contours are 0.10, $0.13,0.16,0.19$, and $0.22 \mathrm{~K}$. $\left[T_{A}(1,1 ; \mathrm{m})\right.$ contours, this paper; $\mathrm{CO}$ bipolar outflow, Snell et al. 1988]. The positional error of the IRAS source is $\sim \pm 20^{\prime \prime}$.

The $\mathrm{H}_{2} \mathrm{O}$ maser, $\mathrm{HHL} 73$, and the $I R A S$ source, spatially coincident within observationals errors, are located in this clump. We did not detect $\mathrm{NH}_{3}(2,2)$ emission toward the peak of this clump (Table 3 ). The second ammonia clump, located $\sim 5^{\prime}$ northeast of the elongated structure, is barely resolved. No signs of star formation associated with this clump are known at present.

In Figure 8 we show a position-velocity diagram along the major axis of the main clump. A small velocity gradient of $\sim 0.5 \mathrm{~km} \mathrm{~s}^{-1} \mathrm{pc}^{-1}$, with the radial velocity decreasing from northwest to southeast, is seen. If this velocity gradient is caused by the rotation of the clump, the mass necessary to gravitationally bind the motions is estimated to be $\sim 10 M_{\odot}$. Since the estimated mass for this condensation is $\sim 500 \mathrm{M}_{\odot}$ (Table 4), we suggest that this structure could be rotating.

Finally, $\mathrm{CO}$ observations could reveal the presence of highvelocity gas associated with this site of star formation.

\section{e) $S 140 N$}

Fukui et al. (1986) detected a CO bipolar outflow with a low degree of collimation. An IRAS source is located at the center of this outflow, suggesting that it is the powering source. Our $\mathrm{NH}_{3}(1,1)$ map was centered at this position. We detected an ammonia condensation slightly elongated in the northeastsouthwest direction (Fig. 9). $\mathrm{NH}_{3}(2,2)$ emission was detected toward the peak of the ammonia core (Fig. 2; Table 3). From these data we estimate a rotational temperature of $T_{R}(2,2 ; 1$, $1) \simeq 15 \mathrm{~K}$, which, as discussed above for AFGL 5157, implies a local heating effect, probably produced by the nearby $I R A S$ source. The high-density structure observed in $\mathrm{NH}_{3}(1,1)$ seems to be spatially associated with the $\mathrm{CO}$ outflow. The ammonia peak is slightly displaced to the east of the outflow center. However, this outflow has been mapped in $\mathrm{CO}$ with a significantly higher angular resolution than in $\mathrm{NH}_{3}$, so ammonia observations with higher resolution are necessary to clarify whether the observed clump plays an important role in the collimation of this $\mathrm{CO}$ outflow.

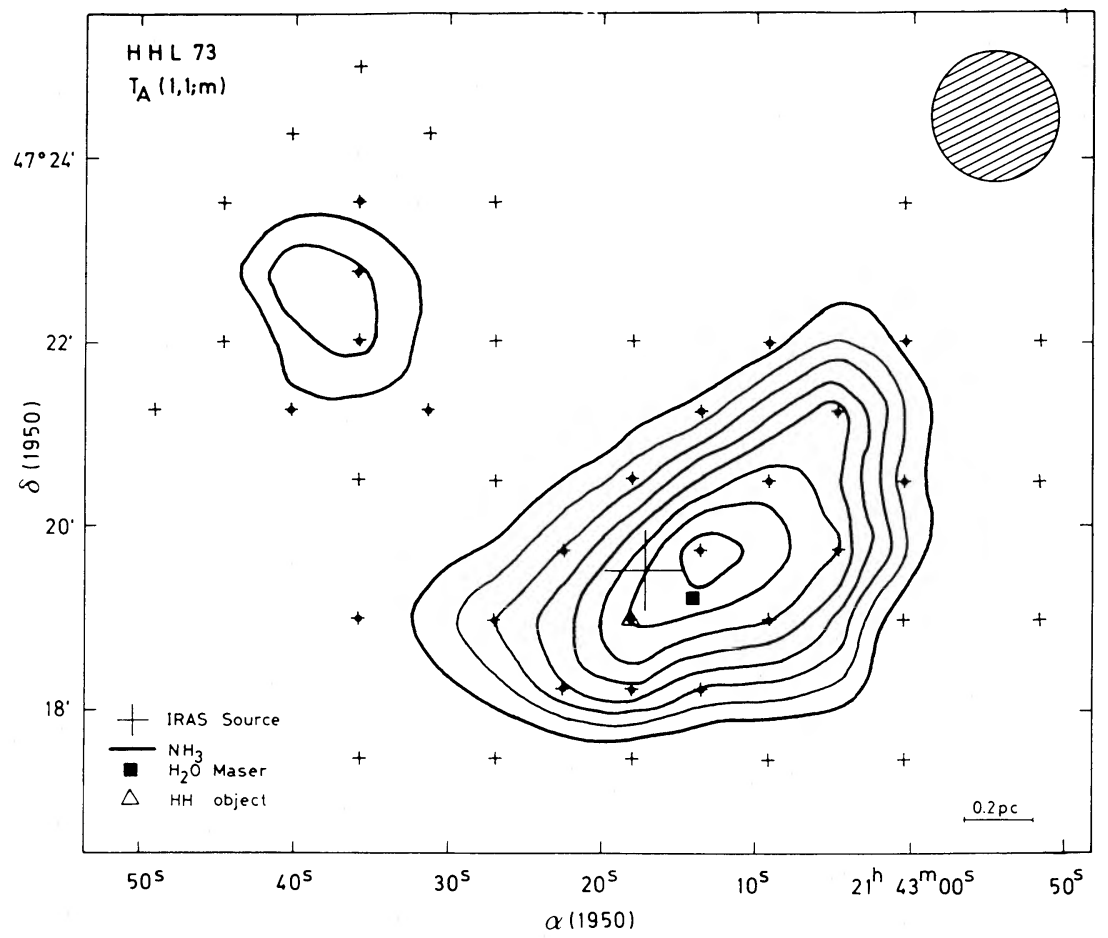

Fig. 7.-Same as Fig. 3, but for HHL 73 . Ammonia contours are $0.12,0.16,0.20,0.28,0.32,0.36,0.40$, and $0.44 \mathrm{~K}$. $\left[T_{A}(1,1 ; \mathrm{m})\right.$ contours, this paper; HHL 73 and $\mathrm{H}_{2} \mathrm{O}$ maser, Gyulbudaghian, Rodríguez, and Mendoza-Torres 1987]. The positional error of the IRAS source is $\sim \pm 20^{\prime \prime}$. 


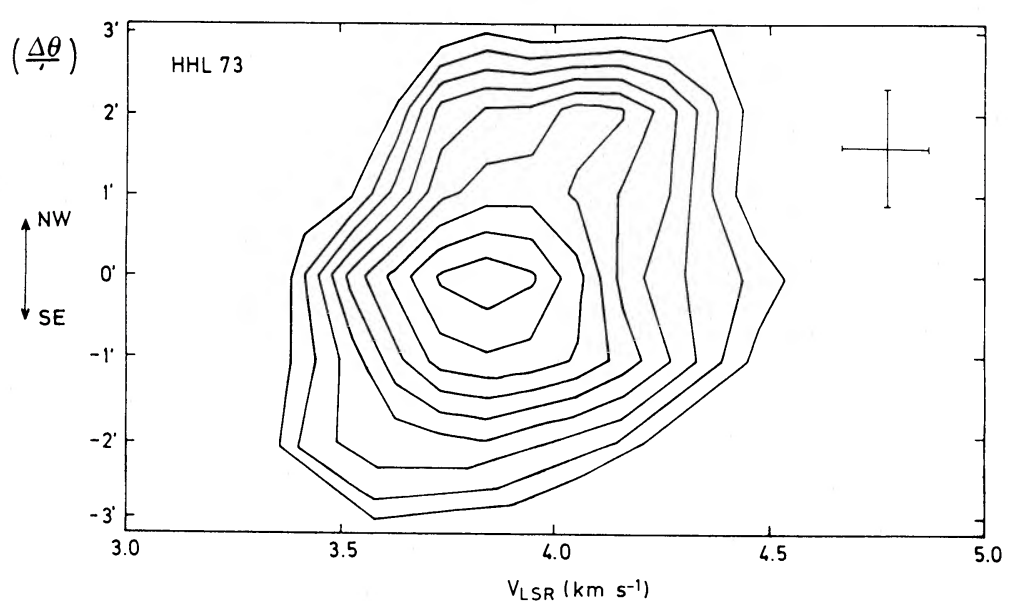

FIG. 8.-Position velocity diagram along the major axis of $\mathrm{HHL} 73$ (p.a. $\left.=-45^{\circ}\right)$. The contour levels of the $(1,1 ; \mathrm{m})$ line are $0.12,0.16,0.20,0.24,0.28,0.32,0.36$, 0.40 , and $0.44 \mathrm{~K}$. This velocity cut shows a velocity gradient of $\sim 0.5 \mathrm{~km} \mathrm{~s}^{-1} \mathrm{pc}^{-1}$. The spatial coordinate (vertical axis) is with respect to the position of the maximum ammonia emission.

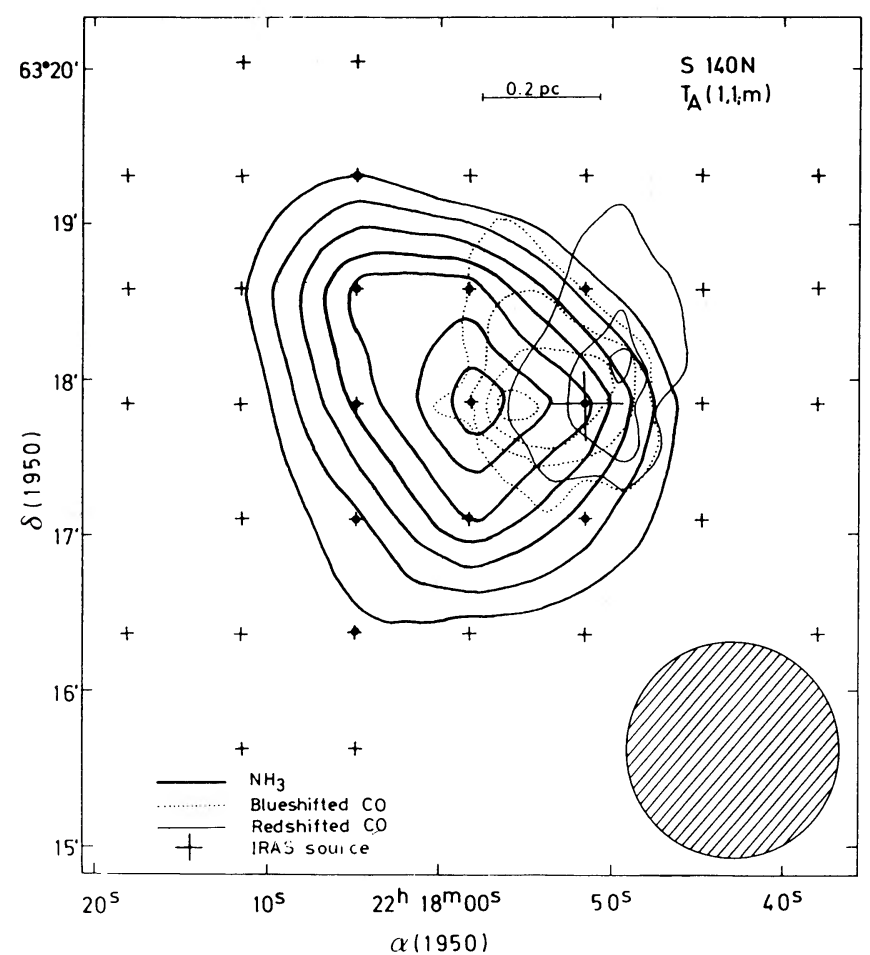

Fig. 9.-Same as Fig. 3, but for S140N. Ammonia contours are 0.12, 0.16, $0.20,0.24,0.28,0.32$, and $0.36 \mathrm{~K}$. [ $T_{A}(1,1 ; \mathrm{m})$ contours, this paper; $\mathrm{CO}$ bipolar outflow, Fukui et al. 1986]. The positional error of the IRAS source is $\sim \pm 20^{\prime \prime}$.

\section{CONCLUSIONS}

We have studied in the $(J, K)=(1,1)$ ammonia transition six regions with star formation signs. We detected and mapped ammonia emission in five of these regions: AFGL 5142, AFGL 5157, AFGL 6366S, HHL 73, and S140N. $\mathrm{NH}_{3}(2,2)$ emission was detected toward the peak of the $\mathrm{NH}_{3}(1,1)$ core of AFGL 5157 and $\mathrm{S} 140 \mathrm{~N}$. We estimate for these two regions a rotational temperature of $T_{R}(2,2 ; 1,1) \sim 16 \mathrm{~K}$. Two new strong $\mathrm{H}_{2} \mathrm{O}$ masers $\left(S_{v} \geq 40 \mathrm{Jy}\right)$ were detected toward the ammonia clumps of AFGL 5142 and AFGL 5157.

The detected high-density structures in AFGL 5142 and AFGL 5157 are barely resolved by our beam $(\sim 1.4)$, and they peak very close to the geometrical center of previously reported bipolar outflows, suggesting that these structures may play a role in the collimation processes of the outflows. However, the infrared source AFGL 5157 appears to be displaced $\sim 1^{\prime}$ southwest of the ammonia peak. A search for near- and farinfrared emission covering the region around the ammonia peak emission- $\mathrm{H}_{2} \mathrm{O}$ maser position could lead to the identification of a better candidate for the powering source of the molecular outflow.

The molecular outflow in AFGL 6366S is located at the southeast edge of the ammonia core. This peculiar spatial disposition could be the result of the interaction of high-velocity gas associated with a young star located at the southeast boundary of the ammonia core. The molecular outflow in S140N is similarly displaced with respect to the molecular core, and may be a similar case to AFGL 6366S. However, the spatial separation is not as certain in this case because of resolution effects.

Finally, we detected an elongated ammonia core in HHL 73 with a small velocity gradient along its major axis. This core peaks near a previously detected IRAS source and an $\mathrm{H}_{2} \mathrm{O}$ maser.

We thank Thomas Kuiper for a careful reading of the manuscript. J. M. T. and L. V. acknowledge partial financial support from Junta de Andalucía, Spain. G. A., R. E., R. L., J. M. T., and L. V. are supported in part by SEUI (Spain), grant PB870371.

\section{REFERENCES}

Acker, A., Chopinet, M., Pottasch, S. R., and Stenholm, B. 1987, Astr. Ap. Suppl., 71, 163 .

Anglada, G., Rodríguez, L. F. Torrelles, J. M., Estalella, R., Ho, P. T. P., Cantó,

J., López, R., and Verdes-Montenegro, L. 1989, Ap. J., 341, 208.

Barral, J. F., and Cantó, J. 1981, Rev. Mexicana Astr. Ap., 5, 101.

Boss, A. 1987, Ap.J., 316, 721.
Cantó, J., Tenorio-Tagle, G., and Różyczka, M. 1988, Astr. Ap., 192, 287.

Cohen, M., and Schwartz, R. D. 1987, Ap. J., 316, 311.

Crampton, D., and Fisher, W. A. 1974, Pub. Dom. Ap. Obs., 14 (No. 12), 283.

Elias, J. H. 1978, Ap. J., 223, 859.

Fukui,Y., Suitni, K., Takaba, H., Iwata, T., Mizuno, A., Ogawa, H., and Kawabata, K. 1986, Ap. J.(Letters), 311, L85. 
Goldsmith, P. F., and Langer, W. D. 1978, Ap. J., 222, 881.

Gyulbudaghian, A. L., Rodríguez, L. F., and Mendoza-Torres, E. 1987, Rev.

Mexicana Astr. Ap., 15, 53.

Hartmann, L., and Kenyon, S. J. 1987, Ap. J., 312, 243.

Herbst, E., and Klemperer, W. 1973, Ap. J., 185, 505.

Heyer, M. H., Snell, R. L., Goldsmith, P. F., Strom, S. E., and Strom, K. M. 1986, Ap. J., 308, 134.

Higgs, L. A. 1971, M.N.R.A.S., 153, 315

Ho, P. T. P., and Townes, C. H. 1983, Ann. Rev. Astr. Ap., 21, 239.

Kaifu, N., et al. 1984, Astr. Ap., 134, 7.

Kawabe, R., et al. 1989, preprint.

Königl, A. 1982, Ap. J., 261, 115.

Kwan, J., and Scoville, N. 1976, Ap. J.(Letters), 210, L39.

Lada, C. J. 1985, Ann. Rev. Astr. Ap., 23, 267.

Levreault, R. M. 1988, Ap. J. Suppl., 67, 283.

Little, L. T., Dent, W. R. F., Heaton, B., Davies, S. R., and White, G. J. 1985, M.N.R.A.S., 217, 227.

Lutz, J. H., and Kaler, J. B. 1983, Pub. A.S.P., 95, 739.

Marcaide, J. M., Torrelles, J. M., Güsten, R., Menten, K. M., Ho, P. T. P.,

Moran, J. M., and Rodríguez, L. F. 1988, Astr. Ap., 197, 235.

Menten, K. M., and Walmsley, C. M. 1985, Astr. Ap., 146, 369.

Moriarty-Schieven, G. H., Snell, R. L., Strom, S. E., and Grasdalen, G. L. 1987 Ap. J.(Letters), 317, L95.

Mundt, R. 1986, Canadian J. Phys., 64, 407

Mundt, R., Bührke, T., Fried, J. W., Neckel, T., Sarcander, M., and Stocke, J. 1984, Astr. Ap. 140, 17.

Myers, P. C., and Benson, P. J. 1983, Ap. J., 266, 309.

Myers, P. C., Heyer, M., Snell, R. L., and Goldsmith, P. F. 1988, Ap. J., 324, 907.

Rodríguez, L. F., Cantó, J., Torrelles, J. M., and Ho, P. T. P. 1986, Ap. J. (Letters), 301, L25.
Rodríguez, L. F., Carral, P., Ho, P. T. P., and Moran, J. M. 1982, Ap. J., 260, 635.

Sabbadin, F., and Hamzaoglu, E. 1981, Astr. Ap., 94, 25.

Sarcander, M., Neckel, T., and Elsässer, H. 1985, Ap. J. (Letters), 288, L51.

Schwartz, P. R., Waak, J. A., and Smith, H. A. 1983, Ap. J. (Letters), 267, L109.

Snell, R. L., Huang, Y.-L., Dickman, R. L., and Claussen, M. J. 1988, Ap. J., $325,853$.

Spitzer, L. 1978, Physical Processes in the Interstellar Medium (New York: Wiley).

Takano, T. 1986, Ap. J., 303, 349.

Takano, T., Stutzki, J., Fukui, Y., and Winnewisser, G. 1987, IAU Symposium 115, Star Forming Regions, ed. M. Peimbert and J. Jugaku (Dordrecht: Reidel), p. 361.

Takano, T., Stutzki, J., Winnewisser, G., and Fukui, Y. 1985, Astr. Ap., 144, L20.

Thronson, H. A., and Lada, C. J. 1984, Ap. J., 284, 135

Torrelles, J. M., Anglada, G., Rodríguez, L. F., Cantó, J., and Barral, J. F. 1987a, Astr. Ap., 177, 171.

Torrelles, J. M., Cantó, J., Rodríguez, L. F., Ho, P. T. P., and Moran, J. M. 1985, Ap. J.(Letters), 294, L117.

Torrelles, J. M., Ho, P. T. P., Moran, J. M., Rodríguez, L. F., and Cantó, J. 1986a, Ap. J., 307, 787.

Torrelles, J. M., Ho, P. T. P., Rodríguez, L. F., and Cantó, J. 1986b, Ap. J., 305, 721.

Torrelles, J. M., Ho. P. T. P., Rodríguez, L. F., Cantó, J., and Moran, J. M. 1987 b, Ap. J., 321, 884.

Torrelles, J. M., Rodríguez, L. F., Cantó, J., Carral, P., Marcaide, J., Moran, J. M., and Ho, P. T. P. 1983, Ap. J., 274, 214.

Zuckerman, B., Kuiper, T. B. H., and Rodríguez Kuiper, E. N. 1976, Ap. J. (Letters), 209, L137.

G. Anglada, R. Estalella, and R. López: Departament de Física de l'Atmosfera, Astronomia i Astrofisica, Universitat de Barcelona, Diagonal 647, E-08028 Barcelona, Spain

J. CANTó and L. F. Rodríguez: Instituto de Astronomía, UNAM, Apdo. Postal 70-264, 04510, México, D.F., Mexico

P. T. P. Ho: Harvard-Smithsonian Center for Astrophysics, 60 Garden Street, Cambridge, MA 02138

J. M. Torrelles and L. Verdes-Montenegro: Instituto de Astrofísica de Andalucía, CSIC, Apdo. Correos 2144, E-18080 Granada, Spain 